\section{A) Check for updates}

Cite this: Nanoscale, 2020, 12, 5922

\title{
Local strain-driven migration of oxygen vacancies to apical sites in $\mathrm{YBa}_{2} \mathrm{Cu}_{3} \mathrm{O}_{7-x^{\dagger}}$
}

\author{
Bernat Mundet, $\$ \S^{\mathrm{a}}$ Steven T. Hartman, (D) $\ddagger^{\mathrm{b}}$ Roger Guzman, ${ }^{\mathrm{a}}$ Juan C. Idrobo, ${ }^{\mathrm{C}}$ \\ Xavier Obradors, ${ }^{a}$ Teresa Puig, ${ }^{a}$ Rohan Mishra (D) *d and Jaume Gázquez (D) *a
}

It is well known that in the high-temperature superconductor $\mathrm{YBa}_{2} \mathrm{Cu}_{3} \mathrm{O}_{7-x}(\mathrm{YBCO})$, oxygen vacancies $\left(V_{O}\right)$ control the carrier concentration, its critical current density and transition temperature. In this work, it is revealed that $V_{O}$ also allows the accommodation of local strain fields caused by large-scale defects within the crystal. We show that the nanoscale strain associated with $\mathrm{Y}_{2} \mathrm{Ba}_{4} \mathrm{Cu}_{8} \mathrm{O}_{16}(\mathrm{Y} 124)$ intergrowthsthat are common defects in $\mathrm{YBCO}-$ strongly affect the venue and concentration of $\mathrm{V}_{\mathrm{O}}$. Local probe measurements in conjunction with density-functional-theory calculations indicate a strain-driven reordering of $\mathrm{V}_{\mathrm{O}}$ from the commonly observed $\mathrm{CuO}$ chains towards the bridging apical sites located in the $\mathrm{BaO}$ plane and bind directly to the superconducting $\mathrm{CuO}_{2}$ planes. Our findings have strong implications on the physical properties of the $\mathrm{YBCO}$, as the presence of apical $\mathrm{V}_{\mathrm{O}}$ alters the transfer of carriers to the $\mathrm{CuO}_{2}$ planes, confirmed by changes in the $\mathrm{Cu}$ and $\mathrm{O}$ core-loss edge probed using electron energy loss spectroscopy, and creates structural changes that affect the $\mathrm{Cu}-\mathrm{O}$ bonds in the superconducting planes. In addition, the revelation of apical $V_{O}$ also has implications on modulating critical current densities and enhancing vortex pinning.

Received 23rd January 2020,

Accepted 17th February 2020

DOI: $10.1039 / \mathrm{d} 0 \mathrm{nr} 00666 \mathrm{a}$

rsc.li/nanoscale studying subtle changes in the structure around defects or at interfaces. The study of $\mathrm{YBa}_{2} \mathrm{Cu}_{3} \mathrm{O}_{7-x}$ (YBCO) illustrates how aberration-corrected STEM reveals complex point defects that had remained invisible to other characterization techniques, explaining the origins of functionalities such as diluted ferromagnetism in YBCO films. ${ }^{1}$

YBCO has excellent superconducting performance at high magnetic fields and temperature conditions well above liquid $\mathrm{N}_{2}$ 's boiling point, thus it is a strong candidate for high current and power applications. ${ }^{14}$ Since the observation of high-temperature superconductivity in YBCO in $1987,{ }^{15}$ there have been numerous reports on the influence of the oxygen in YBCO, either when grown as a single crysta ${ }^{16-20}$ or as a thin film, ${ }^{21-23}$ on its structure and superconducting properties. YBCO's unit cell structure, shown in Fig. 1(a), is an oxygendeficient triple perovskite with two fully-oxygenated $\mathrm{CuO}_{2}$ superconducting planes separated by an oxygen-free $\mathrm{Y}$ layer. ${ }^{20}$ Each $\mathrm{CuO}_{2}$ superconducting plane is connected to a $\mathrm{Cu}-\mathrm{O}$ chain layer that is oriented along the $b$-axis, through a bridging or apical $\mathrm{O}$ - referred to as $\mathrm{O}(4)$ in Fig. 1(a). Hence, orthorhombic YBCO has five inequivalent oxygen sites. Oxygen not only governs the structural parameters of YBCO but also its superconducting properties, as each oxygen dopes additional hole carriers to the $\mathrm{CuO}_{2}$ superconducting planes. ${ }^{24}$ The superconducting critical temperature $\left(T_{\mathrm{C}}\right)$ progressively decreases from a maximum value of $92 \mathrm{~K}$, when the structure has a vacancy concentration of $x=0.07$, down to the suppression of 
(a)

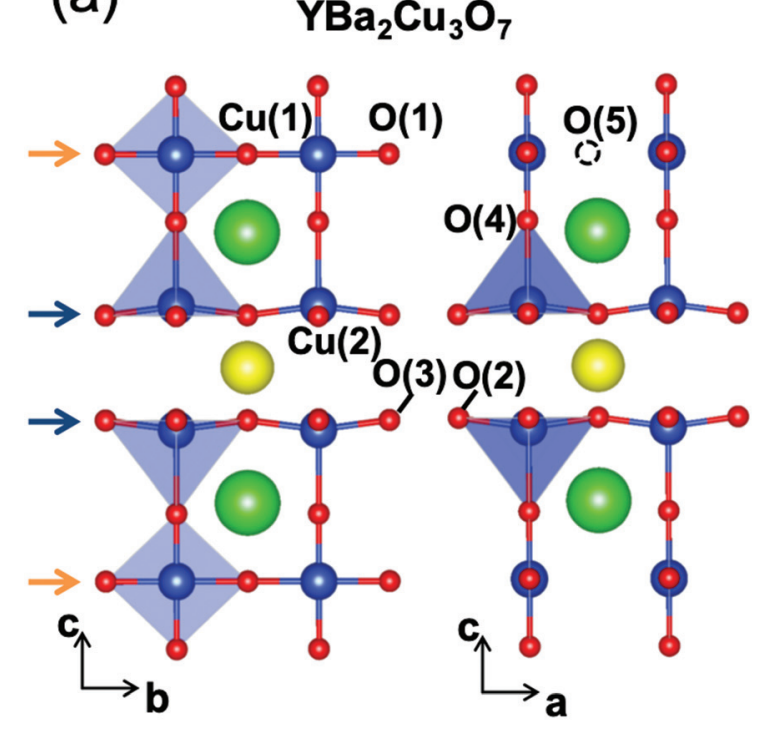

(b)

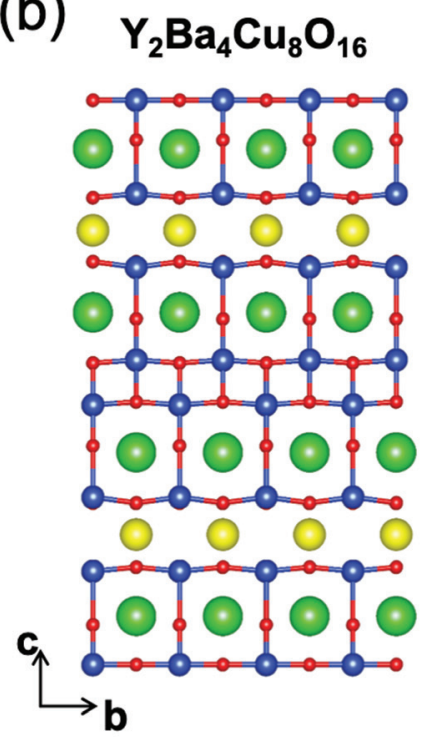

\section{$\mathrm{Ba} \bigcirc \mathrm{Y} \circ \mathrm{Cu} \bullet \mathrm{O}$}

Fig. 1 (a) Illustration of the orthorhombic YBCO unit cell structure viewed along the [100] (left) and [010] (right) zone axis, where the different Cu and $\mathrm{O}$ crystallographic positions are labeled. The blue and orange arrows point to the superconducting planes and chain layers, respectively. (b) Illustration of the Y124 intergrowth viewed along the [100]-zone axis.

superconductivity at $x \approx 0.5$, where the structure becomes tetragonal. ${ }^{17,20,24}$

It is commonly accepted that out of the five inequivalent oxygen sites, vacancies predominantly reside in the $\mathrm{Cu}-\mathrm{O}$ chain layers, herein referred to as $\mathrm{V}_{\mathrm{O}(1)}$. There are a few reports suggesting small concentrations of apical $\mathrm{V}_{\mathrm{O}(4)}$ that are directly connected to the superconducting $\mathrm{CuO}_{2}$ planes. ${ }^{18,25,26}$ Recently, direct evidence of the presence of apical $\mathrm{V}_{\mathrm{O}(4)}$ in optimally doped YBCO single crystals and thin films was shown using aberration-corrected STEM imaging and electron energy loss spectroscopy. ${ }^{27}$ These observations directly followed theoretical predictions made using first-principles densityfunctional-theory (DFT) calculations showing that in the optimal doping region isolated apical $\mathrm{V}_{\mathrm{O}(4)}$ have similar formation energy as chain $\mathrm{V}_{\mathrm{O}(1)}$. The two vacancies are, therefore, expected to be present at similar concentrations. Besides vacancies which introduce carriers to the lattice, the best performing YBCO samples have a complex microstructure including defects such as stacking faults, dislocations, anti-phase boundaries and secondary phases. Such a complex microstructure is intentionally designed as these defects introduce large strains and act as efficient pinning centers to immobilize the quantized vortices, enabling high critical current density. However, the interaction between the newly reported, but widespread, apical $\mathrm{V}_{\mathrm{O}(4)}$ and the strain due to the complex microstructure of YBCO is missing.

In this work, we demonstrate the relationship between $\mathrm{V}_{\mathrm{O}(4)}$ and the commonly observed Y124 stacking fault defect by examination of thin-film and single crystal samples. We have combined high-angle annular dark-field (HAADF) and annular bright field (ABF) imaging modes to explore the structure of YBCO with oxygen vacancies in the BaO plane. Density functional theory (DFT) calculations were carried out to evaluate the stability of the $\mathrm{V}_{\mathrm{O}(4)}$ and to understand the structural mechanisms driving its generation. While the two vacancy types $\left(\mathrm{V}_{\mathrm{O}(1)}\right.$ and $\left.\mathrm{V}_{\mathrm{O}(4)}\right)$ have similar formation energies under standard conditions, our calculations predict that $\mathrm{V}_{\mathrm{O}(4)}$ 's become relatively more favorable when the YBCO unit cell is vertically compressed. Interestingly, atomic-scale STEM probing of the lattice spacings proved that the YBCO unit cells on either side of Y124 intergrowths are compressed vertically. Our results illustrate both the link between the local strain associated to the Y124 intergrowths and the appearance of the oxygen vacancies in the apical sites, and the structural and electronic changes appearing due to the occurrence of $\mathrm{V}_{\mathrm{O}(4)}$.

\section{Experimental}

\section{Sample fabrication}

A $5 \times 5 \mathrm{~mm}^{2}, 250 \mathrm{~nm}$-thick YBCO thin film has been grown onto a $\mathrm{LaAlO}_{3}$ single crystal substrate following the metalorganic decomposition of trifluoracetates route that is described elsewhere. ${ }^{28,29}$ A $0.5 \times 0.5 \mathrm{~mm}^{2}, 30 \mu \mathrm{m}$-thick YBCO single crystal has been fabricated by using the flux-growth technique described in ref. 30. The STEM specimen of the YBCO thin film was prepared by conventional methods, by grinding, dimpling, and Ar ion milling, whereas the STEM specimen of the single crystal was prepared with a Dual Helios Nanolab Focus Ion Beam. 


\section{Image acquisition}

Scanning transmission electron microscopy images were acquired in an aberration-corrected FEI Titan 60-300 microscope operated at $300 \mathrm{kV}$, fitted with a high-brightness fieldemission gun (X-FEG) and a CEOS probe Cs corrector, in an aberration-corrected Nion UltraSTEM100 microscope operated at $100 \mathrm{kV}$ and in a Nion UltraSTEM200 operated at $200 \mathrm{kV}$, both equipped with a nion probe-aberration corrector and a cold-FEG emission gun.

\section{DFT-calculations}

Regarding the defect formation energies and chemical potentials, we have used density functional theory (DFT) as implemented in the VASP package to evaluate the stability of different vacancy configurations containing isolated oxygen vacancies at inequivalent crystallographic sites by estimating and comparing their associated formation energies. After vacancy creation, the atomic positions were fully relaxed while keeping the cell volume fixed. We used a generalized Monkhorst-Pack grid ${ }^{31,32}$ with a minimum real-space distance of $32 \AA$ for relaxations and $60 \AA$ for high-quality static calculations, and a Gaussian smearing with a width of $0.05 \mathrm{eV}$. We have used a $3 \times 3 \times 1$ supercell structure to study isolated vacancies, and apply the PBEsol functional. ${ }^{33}$ The formation energy associated to a specific vacancy configuration ( $\left.E_{\mathrm{Form}}\right)$ is given by the next equation:

$$
E_{\text {Form }}=E_{\text {vacancy }}+\frac{1}{2} \mu_{\mathrm{O}_{2}}-E_{\text {pristine }}
$$

where $E_{\text {pristine }}$ is the energy of the pristine supercell, $E_{\text {vacancy }}$ is the energy associated to the presence of the $\mathrm{V}_{\mathrm{O}}$ and $\mu_{\mathrm{O} 2}$ is the oxygen gas chemical potential.

We do not include any correction for the known overbinding of $\mathrm{O}_{2}$ at the GGA level as our main purpose is to compare different vacancies, and this error affects all vacancy formation energies equally.

In order to study the influence of strain we have evaluated how the $c$-axis strain influences the formation energy associated to both the superconducting planes and chain layers by applying a uniaxial strain from $-6 \%$ (compressive) to $6 \%$ (tensile) along the $c$-axis in $3 \times 3 \times 1$ supercells. We optimized the $a$ and $b$ axes of the pristine cells according to the Poisson effect and their atomic coordinates. We then created an apical or chain $\mathrm{V}_{\mathrm{O}}$ in the deformed cells and optimized the atomiccoordinates keeping cell size and shape constant.

\section{Results and discussion}

The orthorhombic unit cell structure of $\mathrm{YBa}_{2} \mathrm{Cu}_{3} \mathrm{O}_{7}$ is illustrated in Fig. 1(a). The $\mathrm{Cu}$ cations $[\mathrm{Cu}(2)]$ belonging to the superconducting planes (blue arrows) are coordinated with $5 \mathrm{O}$ atoms, forming a squared pyramidal unit, while the $\mathrm{Cu}$ cations $[\mathrm{Cu}(1)]$ of the chain layers (orange arrows) are coordinated with $4 \mathrm{O}$ atoms, all placed within the $b-c$ planes. The doubling of the $\mathrm{CuO}_{x}$ chain layer is the most common defect in YBCO, which gives rise to the well-known Y124 intergrowth, ${ }^{34,35}$ shown in Fig. 1(b). Notice that the two parts of the YBCO structure on either side of the fault are shifted laterally by $1 / 2$ $b$ when viewed along the [100] direction leading to a nonconservative stacking fault with a displacement vector of $\left[\begin{array}{ll}0 & b / 2\end{array}\right.$ $c / 6] \cdot{ }^{36,37} \mathrm{~A}$ double $\mathrm{Cu}-\mathrm{O}$ chain with a finite lateral extent is structurally analogous to a Frank loop dislocation, which is an extrinsic stacking fault surrounded by a partial dislocation, see Fig. S2. $\dagger$ As it will be shown later on, the deformation and strain associated to this defect have strong implications on the surrounding YBCO lattice.

Fig. 2(a) shows the simultaneously acquired atomic-resolution HAADF and ABF images obtained from a solution-derived YBCO thin-film. Lower magnification images of this film are included in the supplemental material. The HAADF imaging mode highlights the heaviest atomic species of the YBCO structure, as the image brightness is proportional to the atomic number of each atomic column, so this mode is also called Z-contrast imaging. ${ }^{38,39}$ We observe the lighter oxygen sub-lattice simultaneously using an ABF detector. ${ }^{40}$ The superconducting planes (blue arrows) and the $\mathrm{CuO}_{x}$ chain layers (orange arrows) are directly distinguished in the HAADF image shown in Fig. 1(c), along with two Y124 intergrowths (green arrows). The presence of this lattice defect is highly desirable as it contributes to the pinning of the magnetic flux vortices and enhances the physical properties of YBCO. ${ }^{37,41-47}$ However, its structure and chemistry is complex, ${ }^{1,48}$ as the system forms $\mathrm{Cu}$ and $\mathrm{O}$ vacancies buried within the very same intergrowth in order to balance the stoichiometry when an extra $\mathrm{CuO}_{x}$ layer is inserted in the YBCO structure. Gazquez et al. reported that the most favorable vacancy configuration was the one containing two $\mathrm{Cu}$ vacancies accompanied with three $\mathrm{O}$ vacancies $\left(2 \mathrm{~V}_{\mathrm{Cu}}+3 \mathrm{~V}_{\mathrm{O}}\right)$, with one $\mathrm{O}$ vacancy located in the upper $\mathrm{CuO}$ chain and two in the lower one, ${ }^{1}$ as illustrated in Fig. 2(b). The DFT-predicted $\mathrm{Y}_{2} \mathrm{Ba}_{4} \mathrm{Cu}_{8} \mathrm{O}_{16}$ (Y248) structure in ref. 1, containing double chain layers instead of single ones, is superimposed onto the $\mathrm{ABF}$ image. Notice that the intensity of the oxygen columns in the lower $\mathrm{CuO}$ chain is less than those from the upper $\mathrm{CuO}$ chain, which is in good agreement with the DFT-based predictions.

A closer look at this contrast-inverted $\mathrm{ABF}$ image reveals subtle changes in the nearby YBCO lattice. The first is that those oxygen columns located within the $\mathrm{BaO}$ plane marked with a red arrow - from now on referred to as $\mathrm{BaO}(1)$ - appear brighter than those belonging to the $\mathrm{BaO}$ plane placed below (cyan arrow) and referred to as $\mathrm{BaO}(2)$. The second is that the oxygen atoms belonging to the upper superconducting planethat are closer to the $\mathrm{BaO}(2)$ plane-are shifted upwards, farther form the $Y$ plane, thus increasing the $\mathrm{Cu}-\mathrm{O}-\mathrm{Cu}$ bond angle and hence reducing the buckling of this $\mathrm{CuO}_{2}$ superconducting plane. The reduced intensity at the oxygen columns of the $\mathrm{BaO}(2)$ planes is ascribed to the presence of oxygen vacancies in this site, and we examine these in more detail in a separate publication. ${ }^{27}$ Remarkably, this uneven distribution of apical oxygen vacancies $\left(\mathrm{V}_{\mathrm{O}(4)}\right)$ extend over several unit cells along the $c$-axis. We have confirmed that the intensity 

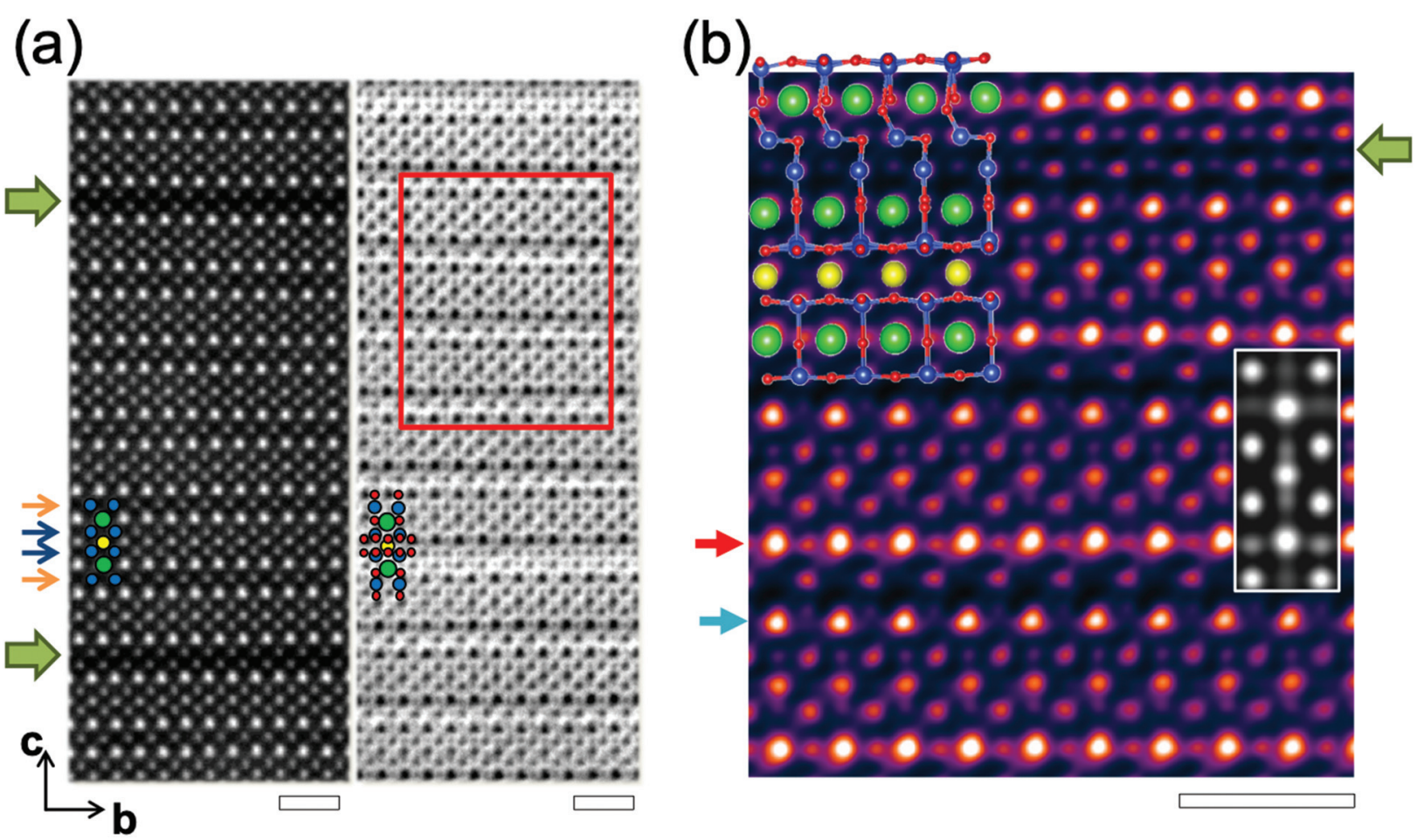

Fig. 2 (a) Z-contrast (left) and ABF (right) images viewed along the [100] zone axis. Blue and orange arrows point to the superconducting planes and chain layers, respectively. An enlarged view of the region marked with the red box in the ABF image is shown in (b) with the contrast inverted and colored. Red and cyan arrows point to a $\mathrm{BaO}(1)$ plane (without $\mathrm{V}_{\mathrm{O}(4)}$ ) and a $\mathrm{BaO}(2)$ plane (with $\mathrm{V}_{\mathrm{O}(4)}$ ), respectively. The superimposed structure corresponds to the DFT-optimized Y248 structure containing several oxygen-decorated pairs of Cu vacancies within the double-chain layer. The inset shows a contrast-inverted ABF image simulation of the $\mathrm{Y} 123$ structure relaxed with $25 \% \mathrm{O}(4)$ vacancies in the $\mathrm{BaO}(2)$ plane. Green arrows point to the Y124 intergrowths. $\mathrm{Ba}, \mathrm{Y}, \mathrm{Cu}$ and $\mathrm{O}$ atoms are represented with green, yellow, blue and red circles, respectively. Scale bars: $1 \mathrm{~nm}$.

reduction agrees well with simulated images of YBCO containing apical vacancies, and we also find that the apical and chain vacancies are equally stable at low concentrations.

While the presence of apical vacancies has been demonstrated recently, there are several open questions regarding their formation. The first is whether these $V_{O}$ 's are generated only in YBCO thin films - that is, are they linked to a certain YBCO fabrication process? The second is whether they are promoted directly by Y124 intergrowths, or by the strain exerted by the substrate? We will answer these questions by investigating the structural properties of a YBCO single crystal (instead of thin films), which we shall describe in the following.

Fig. 3(a) shows a low-magnification HAADF image of a YBCO single crystal. Most of the image presents a homogeneous contrast, indicating the absence of large crystallographic defects. However, within $100 \mathrm{~nm}$ from the crystal's surface, several horizontal dark stripes can be observed. By using high-resolution $Z$-contrast imaging, we have confirmed that these stripes arise due to the presence of Y124 intergrowths (see ESI†े). In addition, a closer look at the YBCO single crystal structure reveals the presence of $V_{O}$ within the $\mathrm{BaO}$ planes in those crystal areas that are rich with Y124 intergrowths. Fig. 3(b) and (c) show contrast-inverted ABF images obtained from two different regions of the single crystal, marked by green and orange squares in Fig. 3(a). In particular,
Fig. 3(b) shows a double $\mathrm{CuO}_{x}$ chain of $\mathrm{Y} 124$ and a contrast variation in the $\mathrm{BaO}$ planes (see the intensity profile shown in Fig. 3(d) like the one shown in Fig. 1(c). Notice that the $O$ columns located within the $\mathrm{BaO}(1)$ planes (red profile) show a larger signal intensity than those located within the $\mathrm{BaO}(2)$ planes (cyan profile). On the other hand, the image acquired from a deeper region far from the Y124 intergrowths (Fig. 3(c)), shows identical $\mathrm{BaO}(1)$ and $\mathrm{BaO} 2(2)$ intensity profiles and is free of any apparent defects. These results provide evidence that $\mathrm{V}_{\mathrm{O}(4)}$ 's and $\mathrm{Y} 124$ intergrowths are unequivocally related, and that the formation of $\mathrm{V}_{\mathrm{O}(4)}$ 's is independent of the synthesis method as we observe them in both thin films and single crystals.

At this stage, another question remains unanswered regarding the driving mechanism favoring the formation of $\mathrm{V}_{\mathrm{O}(4)}$. As the Y124 intergrowths have a finite length, they expand the crystal lattice locally by a sixth of the $c$-axis $(c / 6)$ along the outof-plane direction. We can measure this expansion precisely using a center-of-mass refinement method with picometer resolution to locate each atomic column, as previously shown by Guzman et al. ${ }^{37}$ We have applied this procedure to each unit cell in an area like the one shown in Fig. 4(a), which shows a high-resolution Z-contrast image with two Y124 intergrowths in the center separated by 4 -unit cells. While the lower intergrowth crosses the full image, the upper one begins in the 
(a)

middle of the image (the yellow cross signals the associated partial dislocation). Accordingly, the right side of the image contains one additional $\mathrm{Cu}-\mathrm{O}$ plane, which implies that the system adapts to include the $c / 6$ out-of-plane expansion. We can generate a $c$-axis map, shown in Fig. S4 of the ESI, $\dagger$ where each pixel in the map represents the interplanar distance per atomic row. We have plotted two averaged line profiles in Fig. 4(a), left inset, which extend across either one intergrowth (profile 1 in red) or two intergrowths (profile 2 in cyan). The given values of profile 1 and 2 have been calculated taking 13-unit cells along the basal plane, leading to a statistical error of $0.09 \AA$. The yellow line represents the YBCO out-of-plane parameter $(c=11.68 \AA)$ of the bulk. From these values, we have also calculated their corresponding $c$-axis strain depth-profiles (see Fig. 4(a) right inset), which are obtained from the following expression:

$$
\varepsilon_{\mathrm{c}}=\left(c_{\text {imaged }}-c_{\text {bulk }}\right) / c_{\text {bulk }} \times 100 .
$$

Profile 1 and profile 2 show that far from the Y124 intergrowths the $c$-parameter matches that of bulk YBCO. However, the cells located close to the Y124 intergrowths are significantly compressed, especially in the first unit cell on either side of the intergrowth, with compressive strain values larger than $-1 \%$. This effect is magnified in the area between the two intergrowths (crossed by profile 2), where the compression exceeds $-2 \%$. These results show that Y124 intergrowths induce a local compressive deformation along the $c$-axis. Accordingly, we have calculated how the formation energy of isolated chain $\mathrm{V}_{\mathrm{O}(1)}$ and apical $\mathrm{V}_{\mathrm{O}(4)}$ changes as a function of strain along the $c$-axis. The results are plotted in Fig. 4(b), which shows that for a tensile deformation (positive strain values), both vacancies, $\mathrm{V}_{\mathrm{O}(1)}$ and $\mathrm{V}_{\mathrm{O}(4)}$, are more probable to appear when compared to bulk YBCO (strain-free), as their formation energy is lowered. Yet, as the tensile strain increases, the formation energy of the chain $\mathrm{V}_{\mathrm{O}(1)}$ decreases more rapidly. On the other hand, under a small compressive strain $(<-2 \%)$, the formation energy of both $\mathrm{V}_{\mathrm{O}(1)}$ and $\mathrm{V}_{\mathrm{O}(4)}$ increases, but the increase is weaker for the apical $\mathrm{V}_{\mathrm{O}(4)}$ and it becomes more stable than the $\mathrm{V}_{\mathrm{O}(1)}$. With large compressive strains $\sim-6 \%$, the formation energy of $\mathrm{V}_{\mathrm{O}(4)}$ is lowered that in bulk YBCO. These results suggest that the local compressive strain arising due to Y124 intergrowth increases the formation of apical oxygen vacancies than chain vacancies. 
(a)

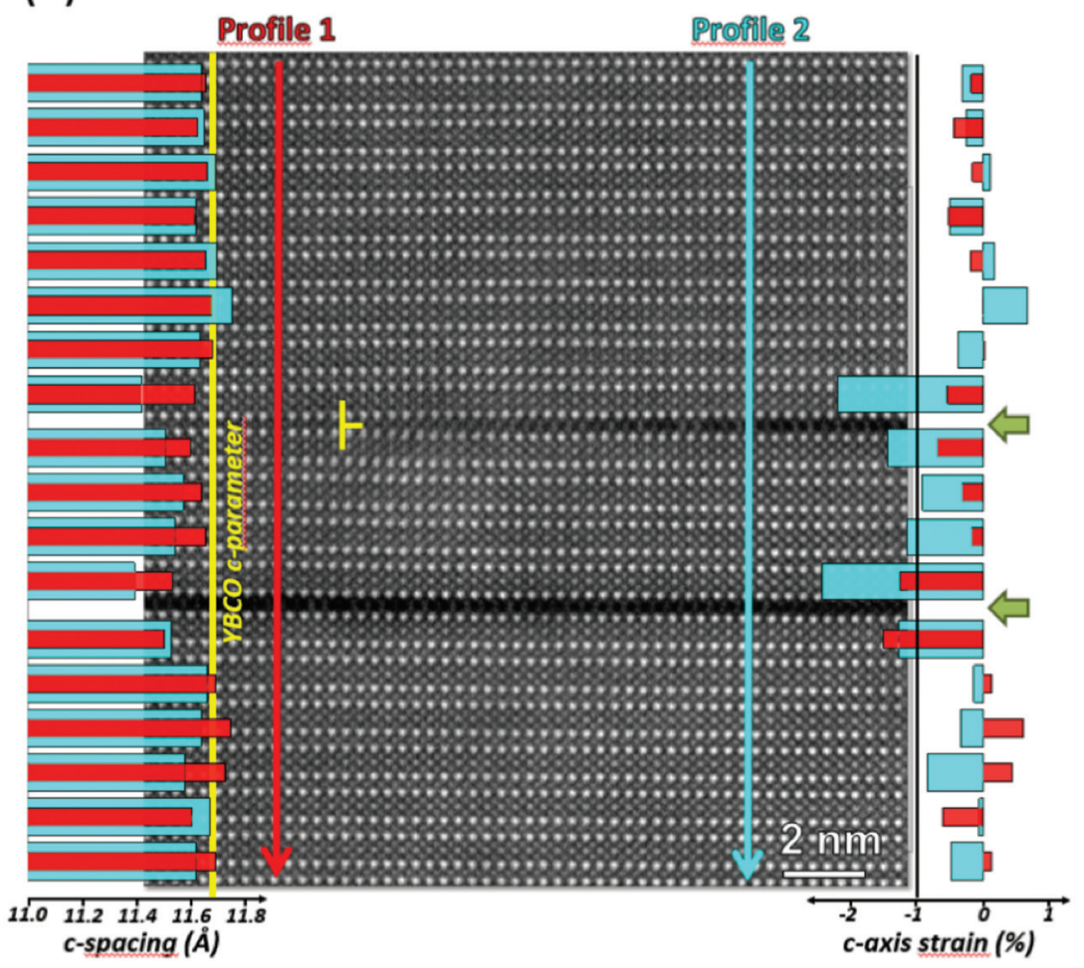

(b)
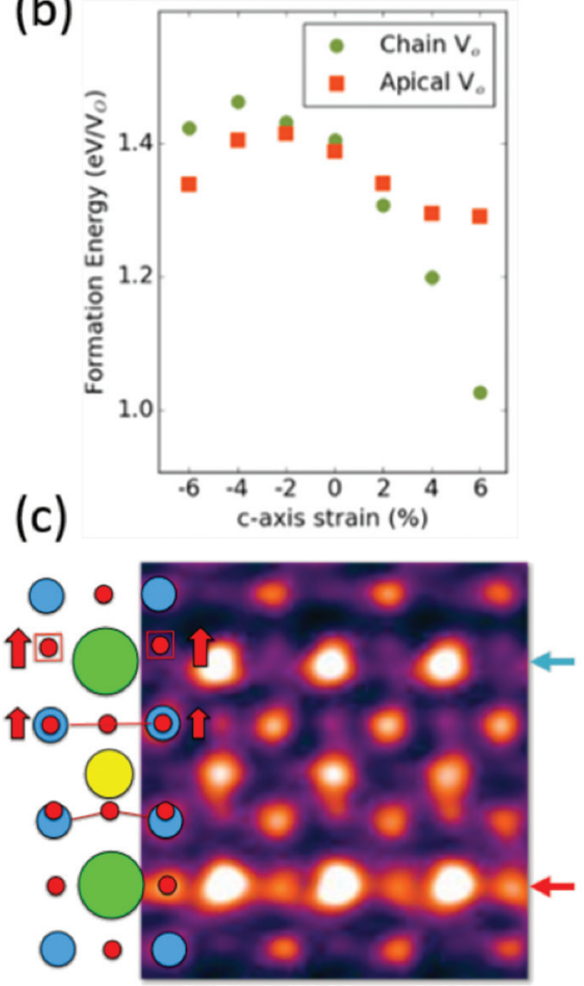

Fig. 4 (a) HAADF image of a YBCO film region containing two Y124 intergrowths (green arrows), one crossing the full image and the other one starting at the middle of the viewed area (yellow cross). Superimposed at the left side of the HAADF image are the c-parameter depth-profiles measured at the left (red vertical arrow) and right (blue vertical arrow) sides of the HAADF image. One of the Ba-Ba spacings located between both intergrowths has been used twice in the calculations, as the crystal periodicity is broken at the planar defect. The yellow line represents the fully relaxed YBCO $c$-axis parameter. Superimposed at the right side of the HAADF image are the depth-profiles of $c$-axis strain calculated from the previous $c$-parameter depth-profiles. (b) DFT-calculated formation energy of one $V_{O}$ located at either the chain (green dots) or apical (orange squares) sites as a function of uniaxial strain along the $c$-axis. Negative/positive strain values represent compressive/tensile uniaxial strains. (c) Illustration of the distorted YBCO unit cell, which is superimposed onto an enlarged image region of the contrast-inverted ABF image shown in Fig. 1(c). Ba, Y, Cu and $O$ atoms are represented with green, blue, yellow and red circles. The red arrows represent the atomic shifts occurring in the oxygen sublattice.

We have used ABF imaging to monitor the picometer-scale lattice distortions occurring within the oxygen sub-lattice near

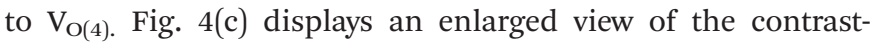
inverted $\mathrm{ABF}$ image that is shown in Fig. 1(c). As sketched in the inset, the oxygen atoms belonging to the upper superconducting plane and apical positions are shifted upwards. On the one hand, these atomic shifts enlarge the spacing between the upper superconducting plane and its corresponding apical oxygen (the one located within the $\mathrm{BaO}(2)$ plane) by $(8 \pm 3) \%$, compared to the same spacing of the superconducting plane that is connected to the fully oxygenated $\mathrm{BaO}(1)$ plane. On the other hand, the oxygen displacements occurring in the upper superconducting plane also increase the $\mathrm{Cu}-\mathrm{O}-\mathrm{Cu}$ buckling angle up to a value of $(176 \pm 2)^{\circ}$ from $(165 \pm 2)^{\circ}$, nearly eliminating the rippling pattern that is commonly displayed by the $\mathrm{Cu}$ and $\mathrm{O}$ atoms when moving along the basal direction in the undistorted superconducting planes.

Using DFT, we have investigated how the combination of compressive strain and apical vacancies affect the atomic structure and electronic properties of YBCO. We show the vari- ation of three key quantities with uniaxial strain along the $c$-axis in Fig. 5: the planar $\mathrm{O}-\mathrm{Cu}-\mathrm{O}$ buckling angle in Fig. 5(a); the in-plane $\mathrm{O}-\mathrm{Cu}$ bond length in Fig. 5(b), and the apical $\mathrm{O}-$ $\mathrm{Cu}$ bond length in Fig. 5(c). All quantities are calculated for the pristine YBCO unit cell and for $3 \times 3 \times 1$ supercells containing a single oxygen vacancy either at the apical or chain site. In the presence of vacancies, the above quantities are measured next to the vacancy. Looking first at the buckling angles in Fig. 5(a), we see that the $\mathrm{CuO}_{2}$ planar unit next to a $\mathrm{V}_{\mathrm{O}(4)}$, which is flatter than normal even without strain, becomes almost perfectly flat (approaching 180 ${ }^{\circ}$ ) under compression. Comparing this relation with that of the pristine YBCO immediately suggests a reason $\mathrm{V}_{\mathrm{O}(4)}$ are preferred under compression. A planar $\mathrm{Cu}$ adjacent to an apical vacancy is underbonded and will tend to move closer to the planar O. This coincides with the strain-induced tendency to reduced buckling of the $\mathrm{CuO}_{2}$ planes, which stabilizes the structure. We note that Poisson's effect leads to an expansion of the planar units under compression, as shown in Fig. 5(b). In principle, this in-plane expansion could make the planar $\mathrm{Cu}$ underbond- 
(a)

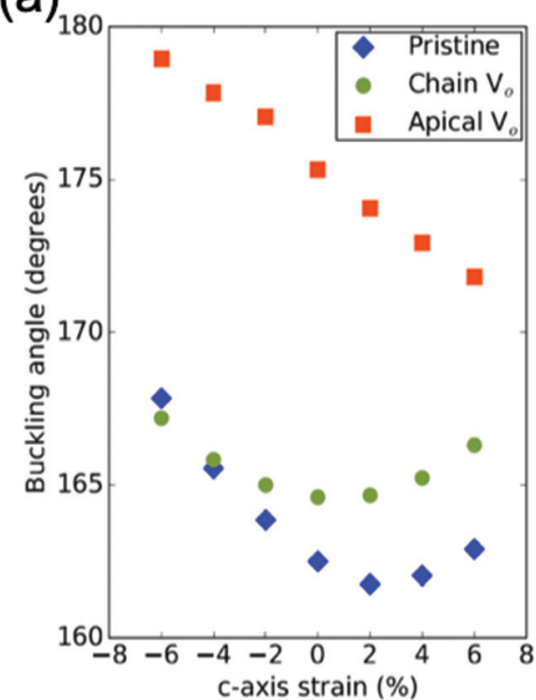

(b)

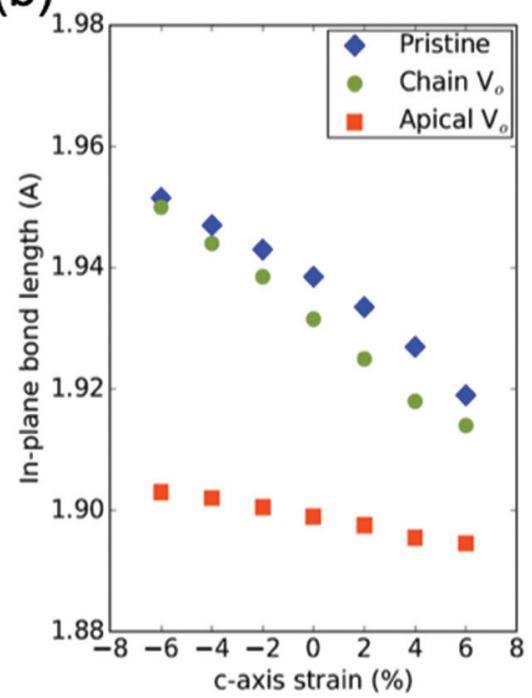

(c)

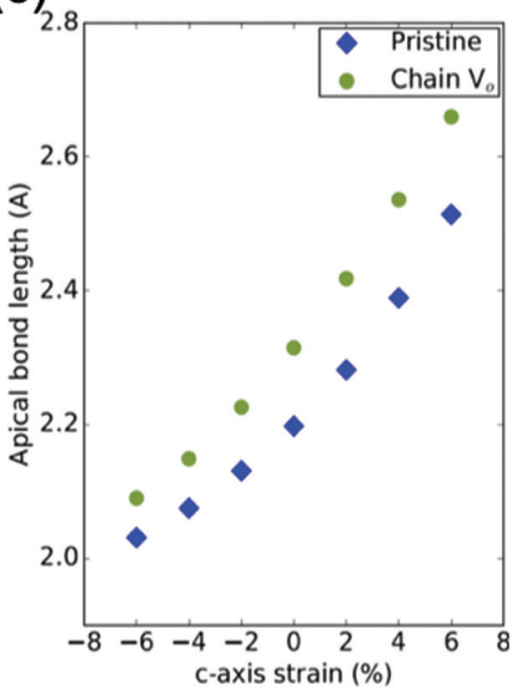

Fig. 5 (a) The in-plane O-Cu-O buckling angle for a 331 DFT-calculated YBCO supercell, as a function of applied strain along the $c$-axis. If the cell contains a chain $\mathrm{V}_{\mathrm{O}}$ (green dot) or apical $\mathrm{V}_{\mathrm{O}}$, the buckling angle is measured in the $\mathrm{CuO}_{2}$ planar unit closest to the vacancy. (b) The planar $\mathrm{Cu} /$ planar $\mathrm{O}$ bond length for the same planar units as (a). (c) The planar $\mathrm{Cu}$ /apical $\mathrm{O}$ bond length for the pristine and chain vacancy structures, again for the same cells as (a).
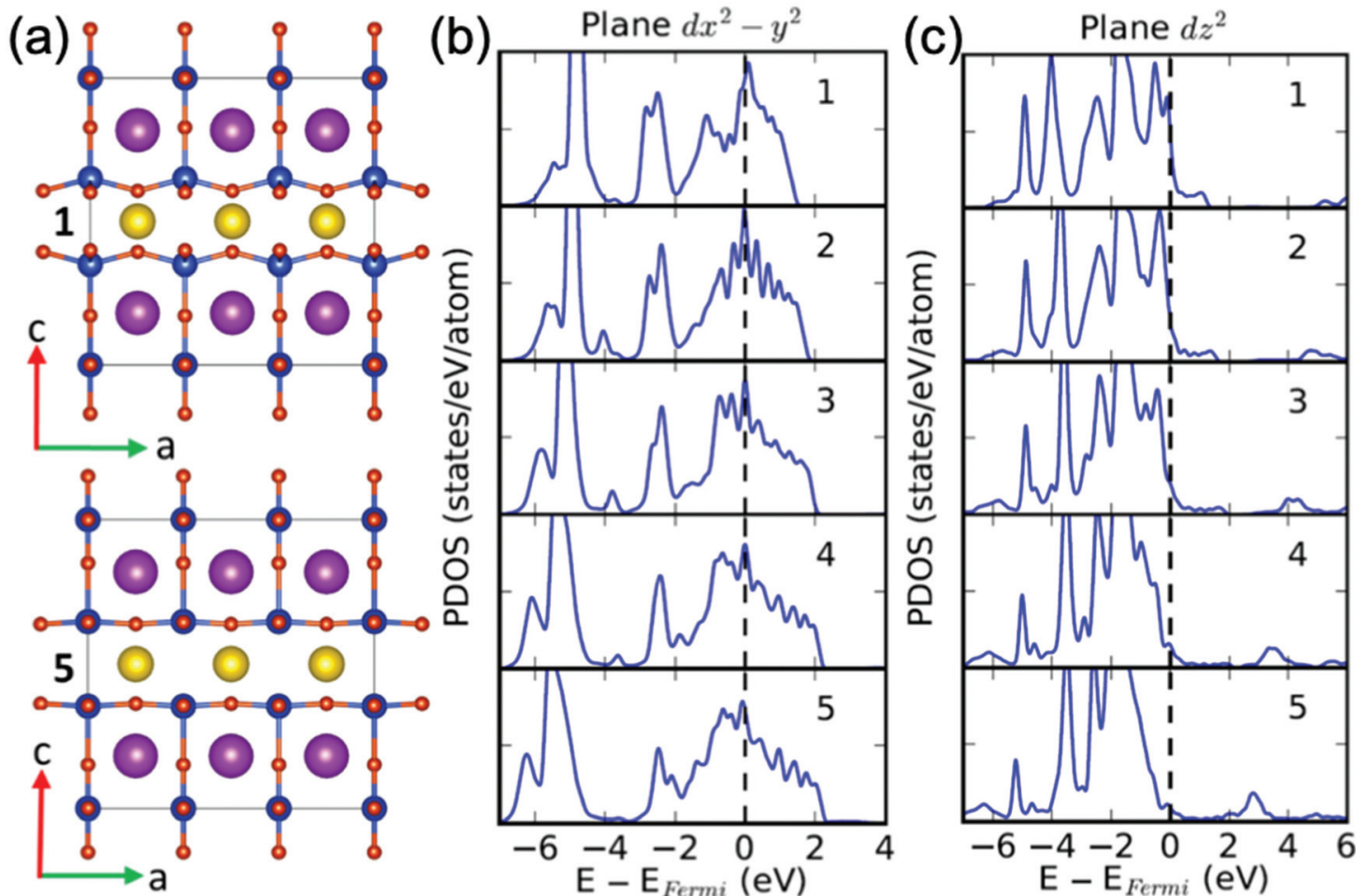

Fig. 6 The effect of a planar buckling mode on the planar copper's electronic density of states in pristine YBCO, projected onto a local orbital basis (PDOS). (a) Five interpolated images of a planar buckling mode in pristine YBCO, with image 1 having maximum buckling and image 5 having completely flat planes. (b) The effect of the buckling mode on the planar copper's electronic density of states, projected onto the $d_{x^{2}-y^{2}}$ orbital. Energies are given relative to the Fermi energy. (c) The projection onto the $d_{z^{2}}$ orbital. 
ing near a $\mathrm{V}_{\mathrm{O}(4)}$ even worse, but Poisson's effect also lengthens the $\mathrm{Cu}-\mathrm{O}$ chains by an equal amount, and so it has little influence on the relative stability of different $\mathrm{V}_{\mathrm{O}}$. Finally, we note that the apical bond length reduction in Fig. 5(c) might destabilize the pristine and chain vacancy structures, but this is not an issue if the apical $\mathrm{O}$ is no longer present.

Finally, we examine the electronic effects of these structural distortions in the presence of both apical vacancies and compressive strain. As shown in Fig. 5(a), the planar buckling is one of the key variables in YBCO which changes in response to structural disturbances, especially apical vacancies. We wish to separate the electronic effects caused directly by the apical vacancy, from those caused indirectly by its effect on the planar buckling, and so in Fig. 6 we show the electronic effects in pristine YBCO of varying one of the phonon modes associated with planar buckling. Image \#5 in Fig. 6(a), with flat $\mathrm{CuO}_{2}$ planes, most closely resembles the apical vacancy structure. There are two main effects; first, as displayed in Fig. 6(b), the planar increases in bandwidth by about $1.5 \mathrm{eV}$ as the planar flattening improves the in-plane orbital overlap. Second, the unfilled band composed of planar $\mathrm{Cu} 4 \mathrm{~s}$, and apical $\mathrm{O}$, which is the "axial orbital" of Pavarini et al. ${ }^{49}$ moves $2 \mathrm{eV}$ closer to the Fermi level and becomes narrower. These effects are due to the motion of the apical $\mathrm{O}$ away from the plane, which is coupled to the motion of the planar $\mathrm{O}$ as part of the buckling mode, and creates effects similar to the presence of an apical vacancy.

We shall compare these changes in the electronic structure with those obtained using electron energy loss spectroscopy with atomic resolution, see Hartman et $a l^{27}$ In this work we gave a detailed description of the subtle spectral changes arising in both the $\mathrm{O} \mathrm{K}$ edge and $\mathrm{Cu} \mathrm{L}$ edge fine structures when comparing the upper superconducting plane, with reduced buckling (adjacent to the apical $\mathrm{V}_{\mathrm{O}}$ ), and the lower one from those YBCO cells neighbouring a Y124 intergrowth defect. The $\mathrm{O} \mathrm{K}$ edge fine structure shows a decrease in the intensity of the pre-peak in the planes with reduced buckling, see ref. 27. As the pre-peak of the O K edge is susceptible to changes in the filling of the unoccupied, hybridized $\mathrm{O}-2 \mathrm{p}$ and $\mathrm{Cu}-3 \mathrm{~d}$ states, ${ }^{50}$ we can ascribe this result to electron doping by the adjacent apical $\mathrm{V}_{\mathrm{O}}$ of the previously unoccupied $\mathrm{O} 2 \mathrm{p}$ states hybridized with $\mathrm{Cu} 3 \mathrm{~d}$ in the superconducting planes and to an enhancement of the in-plane orbital overlap previously shown by the theoretical calculations. ${ }^{27}$ In addition, a second experimental evidence of an electronic reconstruction comes from the annalysis of the $\mathrm{Cu} \mathrm{L}$ edge fine structure, which shows that the intensity of the $\mathrm{Cu} \mathrm{L}_{3}$ edge increases with apical vacancies, see ref. 27.

\section{Conclusions}

We have used the combination of $Z$-contrast and annular bright-field imaging modes to provide a direct observation of the apical oxygen vacancies within the $\mathrm{BaO}$ planes. We have demonstrated that they are present whenever a Y124 inter- growth is formed, either in a YBCO thin film or in a single crystal. Besides, these vacancies display a modulation along the out-of-plane direction, with half of the $\mathrm{BaO}$ planes having many more vacancies. DFT calculations have confirmed the stability of apical vacancies, which is observed to be favored by a compressive strain along the out-of-plane direction, which is exactly the strain effect we observe near Y124 intergrowths. Finally, we have identified additional lattice distortions appearing within the vacancy-rich half of YBCO unit cell, which are expected to influence the YBCO superconducting properties. In particular, we have identified an enlargement of the spacing between the $\mathrm{CuO}_{2}$ superconducting plane and its corresponding apical oxygen, and flattening of the $\mathrm{Cu}-\mathrm{O}-\mathrm{Cu}$ buckling angle.

As the main parameter of interest of YBCO is the critical current density, especially under magnetic fields, the major aim of recent research has been to maximize pinning forces, preventing the dissipative movement of magnetic vortices through the material. The pinning force can be enhanced either by an enriched defect landscape or by increasing the superconducting condensation energy by means of increasing the doping state of YBCO, that is, reaching the overdoped regime. ${ }^{51}$ For that reason, we expect that the presence of oxygen vacancies in apical sites might influence vortex pinning. For instance, $\mathrm{V}_{\mathrm{O}(4)}$ may affect the transfer of holes from the $\mathrm{CuO}_{x}$ chains to the $\mathrm{CuO}_{2}$ superconducting planes and therefore the critical temperature $T_{\mathrm{c}}$. But carrier concentration not only impacts $T_{\mathrm{c}}$, it may also affect the critical current density and vortex pinning in YBCO. ${ }^{51,52}$ In addition, $\mathrm{V}_{\mathrm{O}(4)}$ might also control the carrier concentration when trying to reach the overdoped regime in YBCO through an oxygenation process, since super-oxygenated YBCO forms many intergrowths $^{53}$ and therefore ought to have many apical vacancies as well.

\section{Conflicts of interest}

There are no conflicts to declare.

\section{Acknowledgements}

We acknowledge financial support from the Spanish Ministry of Economy and Competitiveness through the 'Severo Ochoa' Programme for Centres of Excellence in R\&D (SEV-2015-0496), and the COACHSUPENERGY project (SUMATE - RTI2018095853-B-C21, co-financed by the European Regional Development Fund). B. M, R. G., J. G., T. P. and X. O also thank the European Union for its support under the EUROTAPES project (FP7-NMP-Large-2011-280432), ULTRASUPERTAPE project (ERC-2014-ADG-669504), and COST Action NANOCOHYBRI (CA16218), and from the Catalan Government under 2017-SGR-1519 and Xarmae. J. G. also acknowledges the Ramon y Cajal Program (RYC-201211709). S. H. and R. M. acknowledge financial support from 
the National Science Foundation (NSF) grant DMR-1806147. STEM imaging and analysis at $200 \mathrm{kV}$ was sponsored by the U. S. Department of Energy, Office of Science, Basic Energy Sciences, Materials Sciences and Engineering Division, and STEM imaging at $100 \mathrm{kV}$ was conducted at the Center for Nanophase Materials Sciences, which is a DOE Office of Science User Facility. Computations in this work benefited from the use of the Extreme Science and Engineering Discovery Environment (XSEDE), which is supported by NSF grants ACI-1053575 and ACI-1548562.

\section{Notes and references}

1 J. Gazquez, R. Guzman, R. Mishra, E. Bartolomé, J. Salafranca, C. Magén, M. Varela, M. Coll, A. Palau, S. M. Valvidares, P. Gargiani, E. Pellegrin, J. HerreroMartin, S. J. Pennycook, S. T. Pantelides, T. Puig and X. Obradors, Adv. Sci., 2016, 3, 1500295.

2 K. McKenna and A. Shluger, Appl. Phys. Lett., 2009, 95, 222111.

3 R. Mishra, Y.-M. Kim, J. Salafranca, S. K. Kim, S. H. Chang, A. Bhattacharya, D. D. Fong, S. J. Pennycook, S. T. Pantelides and A. Y. Borisevich, Nano Lett., 2014, 14, 2694-2701.

4 Y.-M. Kim, A. Morozovska, E. Eliseev, M. P. Oxley, R. Mishra, S. M. Selbach, T. Grande, S. T. Pantelides, S. V. Kalinin and A. Y. Borisevich, Nat. Mater., 2014, 13, 1019-1025.

5 M. A. Frechero, M. Rocci, G. Sánchez-Santolino, A. Kumar, J. Salafranca, R. Schmidt, M. R. Díaz-Guillén, O. J. Durá, A. Rivera-Calzada, R. Mishra, S. Jesse, S. T. Pantelides, S. V. Kalinin, M. Varela, S. J. Pennycook, J. Santamaria and C. Leon, Sci. Rep., 2015, 5, 17229.

6 R. Guzmán, L. Maurel, E. Langenberg, A. R. Lupini, P. A. Algarabel, J. A. Pardo and C. Magén, Nano Lett., 2016, 16, 2221-2227.

7 R. U. Chandrasena, W. Yang, Q. Lei, M. U. Delgado-Jaime, K. D. Wijesekara, M. Golalikhani, B. A. Davidson, E. Arenholz, K. Kobayashi, M. Kobata, F. M. F. De Groot, U. Aschauer, N. A. Spaldin, X. Xi and A. X. Gray, Nano Lett., 2017, 17, 794-799.

8 J. R. Petrie, C. Mitra, H. Jeen, W. S. Choi, T. L. Meyer, F. A. Reboredo, J. W. Freeland, G. Eres and H. N. Lee, Adv. Funct. Mater., 2016, 26, 1564-1570.

9 J. Gazquez, S. Bose, M. Sharma, M. A. Torija, S. J. Pennycook, C. Leighton and M. Varela, APL Mater., 2013, 1, 012105.

10 S. V. Kalinin and N. A. Spaldin, Science, 2013, 341, 858-859.

11 R. Ishikawa, R. Mishra, A. R. Lupini, S. D. Findlay, T. Taniguchi, S. T. Pantelides and S. J. Pennycook, Phys. Rev. Lett., 2014, 113, 155501.

12 J. H. Jang, Y.-M. Kim, Q. He, R. Mishra, L. Qiao, M. D. Biegalski, A. R. Lupini, S. T. Pantelides, S. J. Pennycook, S. V. Kalinin and A. Y. Borisevich, ACS Nano, 2017, 11, 6942-6949.
13 R. Mishra, R. Ishikawa, A. R. Lupini and S. J. Pennycook, MRS Bull., 2017, 42, 644-652.

14 D. Larbalestier, A. Gurevich, D. M. Feldmann and A. Polyanskii, Nature, 2001, 414, 368-377.

15 M. K. Wu, J. R. Ashburn, C. J. Torng, P. H. Hor, R. L. Meng, L. Gao, Z. J. Huang, Y. Q. Wang and C. W. Chu, Phys. Rev. Lett., 1987, 58, 908-910.

16 J. D. Jorgensen, B. W. Veal, W. K. Kwok, G. W. Crabtree, A. Umezawa, L. J. Nowicki and A. P. Paulikas, Phys. Rev. B: Condens. Matter Mater. Phys., 1987, 36, 5731-5734.

17 R. J. Cava, B. Batlogg, C. H. Chen, E. A. Rietman, S. M. Zahurak and D. Werder, Phys. Rev. B: Condens. Matter Mater. Phys., 1987, 36, 5719-5722.

18 J. D. Jorgensen, H. Shaked, D. G. Hinks, B. Dabrowski, B. W. Veal, A. P. Paulikas, L. J. Nowicki, G. W. Crabtree, W. K. Kwok, L. H. Nunez and H. Claus, Phys. C, 1988, 153-155, 578-581.

19 J. D. Jorgensen, S. Pei, P. Lightfoor, H. Shi, A. P. Paulikas and B. W. Veal, Phys. C, 1990, 167, 571-578.

20 J. D. Jorgensen, B. W. Veal, A. P. Paulikas, L. J. Nowicki, G. W. Crabtree, H. Claus and W. K. Kwok, Phys. Rev. B: Condens. Matter Mater. Phys., 1990, 41, 1863-1877.

21 C. B. Eom, J. Z. Sun, K. Yamamoto, A. F. Marshall, K. E. Luther, T. H. Geballe and S. S. Laderman, Appl. Phys. Lett., 1989, 55, 595-597.

22 Y. M. Boguslavsky and A. P. Shapovalov, Supercond. Sci. Technol., 1991, 4, 149-152.

23 A. P. Shapovalov, Y. M. Boguslavskij, A. I. Ruban, G. G. Gridneva, V. S. Melnikov and N. P. Pshentsova, Supercond. Sci. Technol., 1992, 5, 283-289.

24 J. M. Tranquada, S. M. Heald, A. R. Moodenbaugh and Y. Xu, Phys. Rev. B: Condens. Matter Mater. Phys., 1988, 38, 8893-8899.

25 J. L. MacManus-Driscoll, J. A. Alonso, P. C. Wang, T. H. Geballe and J. C. Bravman, Phys. C, 1994, 232, 288-308.

26 S. H. Pan, E. W. Hudson and J. C. Davis, Rev. Sci. Instrum., 1999, 70, 1459-1463.

27 S. T. Hartman, B. Mundet, J.-C. Idrobo, X. Obradors, T. Puig, J. Gázquez and R. Mishra, Phys. Rev. Mater., 2019, 3, 114806.

28 T. Puig, J. C. González, A. Pomar, N. Mestres, O. Castaño, M. Coll, J. Gázquez, F. Sandiumenge, S. Piñol and X. Obradors, Supercond. Sci. Technol., 2005, 18, 1141-1150.

29 K. Zalamova, N. Romà, A. Pomar, S. Morlens, T. Puig, J. Gázquez, A. E. Carrillo, F. Sandiumenge, S. Ricart, N. Mestres and X. Obradors, Chem. Mater., 2006, 18, 58975906.

30 J. P. Rice and D. M. Ginsberg, J. Cryst. Growth, 1991, 109, 426-431.

31 P. Wisesa, K. A. McGill and T. Mueller, Phys. Rev. B, 2016, 93, 155109.

32 H. J. Monkhorst and J. D. Pack, Phys. Rev. B: Solid State, 1976, 13, 5188-5192.

33 J. P. Perdew, A. Ruzsinszky, G. I. Csonka, O. A. Vydrov, G. E. Scuseria, L. A. Constantin, X. Zhou and K. Burke, Phys. Rev. Lett., 2008, 100, 136406. 
34 H. W. Zandbergen, R. Gronsky and G. Thomas, Phys. Status Solidi, 1988, 105, 207-218.

35 H. W. Zandbergen, R. Gronsky, K. Wang and G. Thomas, Nature, 1988, 331, 596-599.

36 J. Tafto, M. Suenaga and R. L. Sabatini, Appl. Phys. Lett., 1988, 52, 667-668.

37 R. Guzman, J. Gazquez, B. Mundet, M. Coll, X. Obradors and T. Puig, Phys. Rev. Mater., 2017, 1, 024801.

38 S. J. Pennycook and D. E. Jesson, Ultramicroscopy, 1991, 37, 14-38.

39 P. D. Nellist and S. J. Pennycook, Ultramicroscopy, 1999, 78, 111-124.

40 R. Ishikawa, E. Okunishi, H. Sawada, Y. Kondo, F. Hosokawa and E. Abe, Nat. Mater., 2011, 10, 278-281.

41 J. Gutiérrez, A. Llordés, J. Gázquez, M. Gibert, N. Romà, S. Ricart, A. Pomar, F. Sandiumenge, N. Mestres, T. Puig and X. Obradors, Nat. Mater., 2007, 6, 367-373.

42 A. Llordés, A. Palau, J. Gázquez, M. Coll, R. Vlad, A. Pomar, J. Arbiol, R. Guzmán, S. Ye, V. Rouco, F. Sandiumenge, S. Ricart, T. Puig, M. Varela, D. Chateigner, J. Vanacken, J. Gutiérrez, V. Moshchalkov, G. Deutscher, C. Magen and X. Obradors, Nat. Mater., 2012, 11, 329-336.

43 Z. Pingxiang, Z. Lian, J. Ping, B. Weimin, W. Xiaozu and L. Zuhan, Phys. C, 1994, 235-240, 2733-2734.

44 S. I. Kim, F. Kametani, Z. Chen, A. Gurevich, D. C. Larbalestier, T. Haugan and P. Barnes, Appl. Phys. Lett., 2007, 90, 252502.
45 Y. Yamada, K. Takahashi, H. Kobayashi, M. Konishi, T. Watanabe, A. Ibi, T. Muroga, S. Miyata, T. Kato, T. Hirayama and Y. Shiohara, Appl. Phys. Lett., 2005, 87, 1-3.

46 V. Rouco, A. Palau, R. Guzman, J. Gazquez, M. Coll, X. Obradors and T. Puig, Supercond. Sci. Technol., 2014, 27, 125009.

47 P. Mele, R. Guzman, J. Gazquez, T. Puig, X. Obradors, S. Saini, Y. Yoshida, M. Mukaida, A. Ichinose and K. Matsumoto, Supercond. Sci. Technol., 2015, 28, 24002.

48 R. Ramesh, T. S. Ravi, D. M. Hwang, A. Inam, X. X. Xi, X. D. Wu, T. Venkatesan and R. Kilaas, Mater. Lett., 1990, 10, 23-27.

49 E. Pavarini, I. Dasgupta, T. Saha-Dasgupta, O. Jepsen and O. K. Andersen, Phys. Rev. Lett., 2001, 87, 047003.

50 N. Gauquelin, D. G. Hawthorn, G. A. Sawatzky, R. X. Liang, D. A. Bonn, W. N. Hardy and G. A. Botton, Nat. Commun., 2014, 5, 4275.

51 G. Deutscher, APL Mater., 2014, 2, 096108.

52 E. F. Talantsev, N. M. Strickland, S. C. Wimbush, J. G. Storey, J. L. Tallon and N. J. Long, Appl. Phys. Lett., 2014, 104, 242601.

53 H. Zhang, N. Gauquelin, C. McMahon, D. G. Hawthorn, G. A. Botton and J. Y. T. Wei, Phys. Rev. Mater., 2018, 2, 033803. 